\title{
Successful Multidisciplinary Treatment for Aggressive Primary Pulmonary Undifferentiated Pleomorphic Sarcoma
}

\author{
Mitsunori Higuchia Hitoshi Yamadab Kakeru Machino ${ }^{c} \quad$ Ikuro Oshibe $^{c}$ \\ Nobutoshi Soeta ${ }^{c}$ Takuro Saito $^{c}$ Hidetaka Uramoto $^{d}$ Sohsuke Yamada $^{\mathrm{e}}$ \\ Hiroshi Hojo ${ }^{f}$ Hiroyuki Suzukig \\ aDepartment of Thoracic Surgery, Aizu Medical Center, Fukushima Medical University, \\ Aizuwakamatsu, Japan; bepartment of Musculoskeletal Oncology and Metabolic \\ Bone Disease Research, Fukushima Medical University School of Medicine, Fukushima, \\ Japan; 'Department of Surgery, Aizu Medical Center, Fukushima Medical University, \\ Aizuwakamatsu, Japan; dDepartment of Thoracic Surgery, Kanazawa Medical University \\ School of Medicine, Kanazawa, Japan; 'Department of Clinical Pathology, Kanazawa Medical \\ University School of Medicine, Kanazawa, Japan; fDepartment of Pathology, Aizu Medical \\ Center, Fukushima Medical University, Aizuwakamatsu, Japan; 9Department of Chest \\ Surgery, Fukushima Medical University School of Medicine, Fukushima, Japan
}

\section{Keywords}

Undifferentiated pulmonary sarcoma - Malignant fibrous histiocytoma - Multidisciplinary treatment · Long-term complete response

\begin{abstract}
Undifferentiated pleomorphic sarcoma (UPS) was previously known as malignant fibrous histiocytoma (MFH). This sarcoma occurs preferentially in the extremities and retroperitoneal space; primary pulmonary UPS/MFH is rare. We report a 52-year-old woman referred to our hospital with dyspnea and severe cough. Chest computed tomography (CT) revealed a pulmonary mass in the left upper lobe and pleural effusion. Cytology of the effusion showed no malignancy; however, the tumor increased rapidly in size, and the patient's respiratory symptoms worsened. The tumor occupied almost all of the left upper lobe and involved the adjacent pericardium. She underwent left upper lobectomy with pericardial resection and reconstruction. Postoperative pathology of the resected specimen showed undifferentiated pulmonary sarcoma, pT4N0M1a stage IV A, and genetic analyses revealed the V-Ki-ras2 Kirsten rat sarcoma viral oncogene homolog (KRAS) mutation. The patient's dyspnea recurred 1 month postoperatively, and CT showed marked pleural effusion. An 18F-fluorodeoxyglucose positron emission tomography demonstrated abnormal diffuse accumulation of 18F-fluoro-
\end{abstract}


Higuchi et al.: Successful Treatment for Primary Pulmonary Undifferentiated

deoxyglucose in the left pleural cavity. We initiated five cycles of chemotherapy with doxorubicin and ifosfamide, and the patient has been well without recurrence for 24 months after multidisciplinary treatment with surgery followed by systemic combination chemotherapy. We successfully treated our patient with primary pulmonary UPS/MFH using a multidisciplinary approach, even though this sarcoma carries a poor prognosis and is insensitive to both chemotherapy and radiotherapy.

(C) 2020 The Author(s).

Published by S. Karger AG, Basel

\section{Introduction}

Undifferentiated pleomorphic sarcoma (UPS) was previously known as malignant fibrous histiocytoma (MFH), which was first reported by O'Brien et al. in 1964 [1]. MFH did not fit any of the recognized sarcoma categories by the end of the 1980s; however, MFH subsequently became to be the single largest category of sarcoma [2]. In 2013, MFH was declassified as a formal diagnostic entity and renamed "undifferentiated pleomorphic sarcoma" by the World Health Organization. UPS/MFH occurs in the extremities in 16\% and retroperitoneum in $68 \%$ of patients [2]. Primary pulmonary UPS/MFH is uncommon and highly malignant, and there are no optimal or consensus treatment strategies. We experienced a patient with primary pulmonary UPS who achieved long-term complete response with surgery followed by chemotherapy. We also performed next-generation DNA sequencing of the UPS/MFH tissue from this patient.

\section{Case Presentation}

A 52-year-old woman was referred to our hospital because of dyspnea and severe cough. Chest computed tomography at her first visit revealed a pulmonary mass in the left upper lobe with pleural effusion (Fig. 1a). Cytology of the effusion showed no malignancy; however, only 1 month after her visit, the tumor had increased rapidly in size (Fig. 1b) and her respiratory symptoms had worsened. At that time, the tumor occupied almost all of the left upper lobe and involved the adjacent pericardium. She underwent left upper lobectomy with pericardial resection and reconstruction. We confirmed localized pleural dissemination during surgery, which was proven pathologically. The resected specimen measured $15 \mathrm{~cm}$ (maximum size), and postoperative pathology of the specimen showed a diffuse proliferation of admixed spindle-shaped and circular, highly atypical cells, arranged in a characteristic storiform growth pattern (Fig. 2a). Immunohistochemical staining revealed that tumor cells were positive for vimentin (Fig. 2b) and negative for epithelial markers. According to these findings, she was diagnosed as having UPS, pT4N0M1a stage IV A. Genetic analyses by next-generation DNA sequencing revealed a positive v-Ki-ras2 Kirsten rat sarcoma viral oncogene homolog (KRAS) mutation (G12D) at codon 12; programmed death ligand 1 (PD-L1) expression was less than $1 \%$. One month postoperatively, repeat computed tomography revealed marked pleural effusion, and the patient again experienced dyspnea. Postoperative 18F-fluorodeoxyglucose positron emission tomography demonstrated abnormal and diffuse accumulation of 18F-fluorodeoxyglucose in the left pleural cavity (Fig. 1c). We initiated five cycles of chemotherapy with doxorubicin and ifosfamide, which induced grade 3 myelosuppression and febrile neutropenia, as adverse events. The patient has been well without recurrence for 24 months after multidisciplinary treatment with surgery followed by systemic combination chemotherapy (Fig. 1d). 


\section{Case Reports in \\ Oncology}

\begin{tabular}{l|l}
\hline Case Rep Oncol 2020;13:385-391 \\
\hline DOI: 10.1159/000506897 & $\begin{array}{l}\text { ○ 2020 The Author(s). Published by S. Karger AG, Basel } \\
\text { www.karger.com/cro }\end{array}$ \\
\hline
\end{tabular}

Higuchi et al.: Successful Treatment for Primary Pulmonary Undifferentiated Pleomorphic Sarcoma
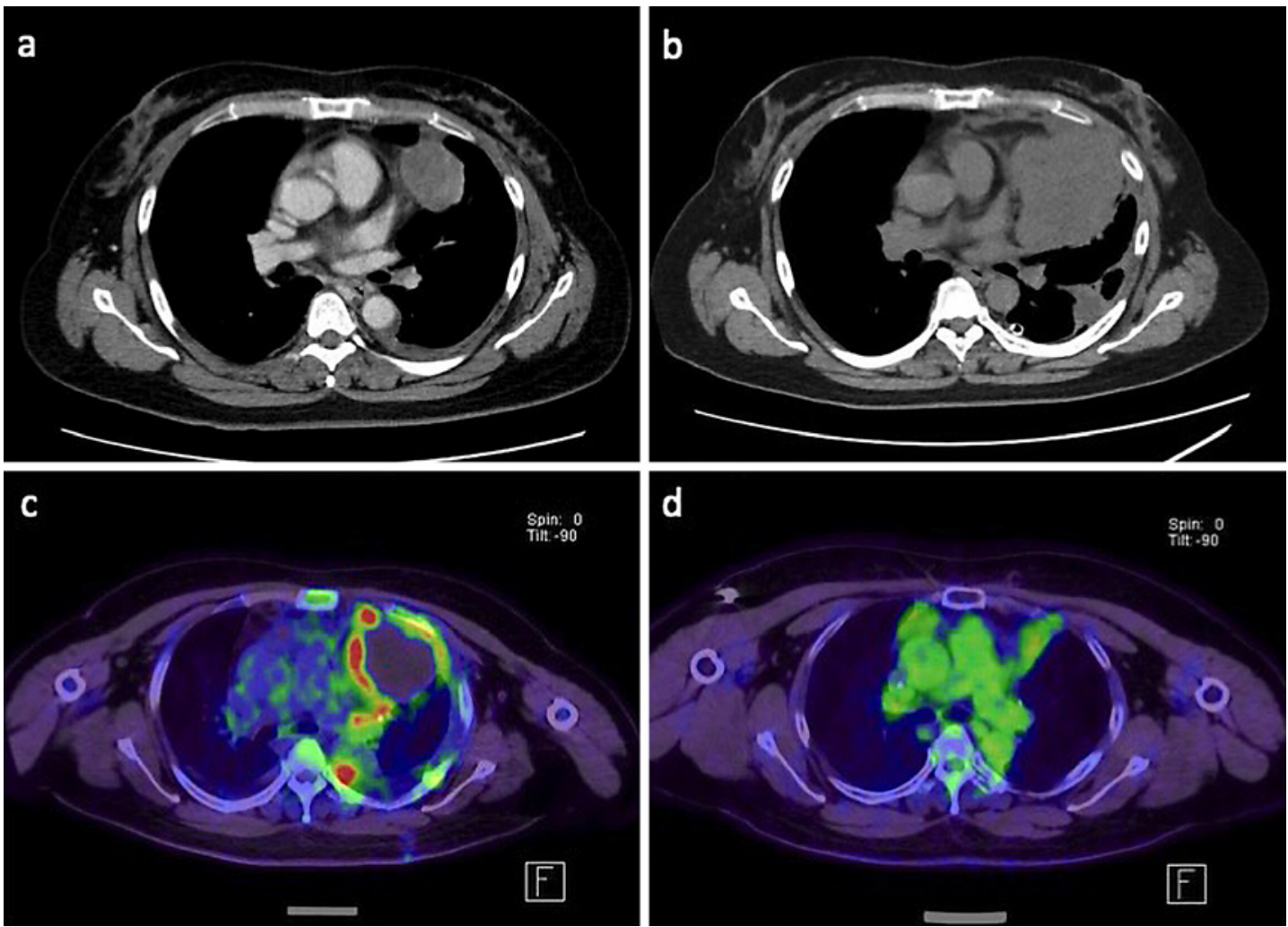

Fig. 1. Chest computed tomographic images showing a pulmonary mass in the left upper lobe with pleural effusion at the patient's first visit (a). This tumor grew rapidly to occupy the entire left upper lobe 1 month after the patient's first visit (b). Postoperative 18F-fluorodeoxyglucose positron emission tomographic images showing abnormal and diffuse accumulation of 18F-fluorodeoxyglucose in the left pleural cavity (c), which disappeared after five cycles of chemotherapy (d).
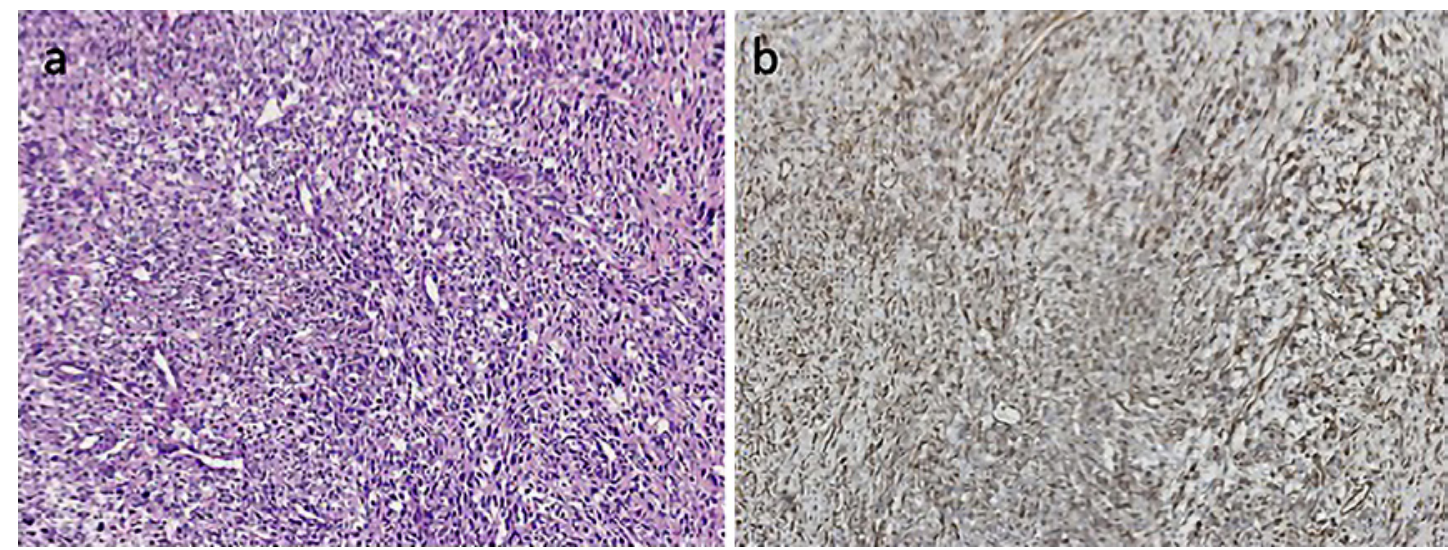

Fig. 2. Histopathological findings showing an admixture of spindle-shaped and circular cells, arranged in a storiform pattern (hematoxylin and eosin, $\times 100$ ) (a). Immunohistochemical staining revealed cells positive for vimentin $(\times 100)(\mathbf{b})$. 
Higuchi et al.: Successful Treatment for Primary Pulmonary Undifferentiated Pleomorphic Sarcoma

Table 1. Patients' characteristics $(n=54)$

\begin{tabular}{lc}
\hline Characteristics & \\
\hline Age, years & $56.1 \pm 15.6$ \\
Sex & \\
$\quad$ Male & $33(61.1 \%)$ \\
Female & $21(38.9 \%)$ \\
Side & \\
$\quad$ Right & $28(51.9 \%)$ \\
Left & $25(46.3 \%)$ \\
Both & $1(1.8 \%)$ \\
Tumor size, cm & $7.2 \pm 4.1$ \\
Treatment & \\
Surgery alone & $37(58.5 \%)$ \\
Surgery and chemotherapy & $3(5.6 \%)$ \\
Surgery and radiotherapy & $6(11.1 \%)$ \\
Surgery, chemotherapy, and radiotherapy & $2(3.7 \%)$ \\
Chemotherapy alone & $1(1.8 \%)$ \\
Radiotherapy alone & $2(3.7 \%)$ \\
No treatment & $3(5.6 \%)$ \\
Nodal status & $12(22.2 \%)$ \\
Positive & $42(77.8 \%)$ \\
Negative & $28(51.9 \%)$ \\
Prognosis & $26(48.1 \%)$ \\
Dead & \\
Alive & \\
\hline
\end{tabular}

\section{Discussion}

UPS/MFH is an aggressive soft tissue sarcoma originating from mesenchymal cells. UPS/ MFH accounts for $5-10 \%$ of sarcomas in adults older than 40 years of age [3]; however, the sarcoma accounts for only $0.04-0.2 \%$ of pulmonary tumors [4]. The symptoms of primary pulmonary UPS/MFH are chest pain, dyspnea, cough, hemoptysis, and weight loss. Our patient also suffered from dyspnea and severe cough. Histologically, tumors are composed of a storiform arrangement of highly pleomorphic and spindle-shaped cells. UPS/MFH shows no specific immunohistochemical findings that enable more specific subclassification [5], and cells stain positive only for vimentin, in most patients, as in our patient. Qorbani et al. [6] reported a brief review of literature on primary pulmonary UPS/MFH of 85 patients who had been reported in the English literature since 1979. We added two reports [7, 8] and our patient to this previous report. 54 out of 88 patients included the complete data of age, gender, location of the tumor, tumor size, treatments, lymph node status, survival time and prognosis. Then we summarized and analyzed the data of primary pulmonary UPS/MFH of 54 patients. These patients' characteristics are shown in Table 1. Survival probabilities were estimated using the Kaplan-Meier method. A $p$ value of $<0.05$ was considered statistically significant. Statistical analysis was conducted with SPSS version 21.0 (IBM Corp., Armonk, NY, USA). 33 out of 54 patients were male and 21 were female. The patient age ranged from 12 to 86 years with a mean age of 56.1 years. The locations of tumor were right side in 28 patients, left side in 25 patients, and both sides in one patient. The tumor size ranged from 1.7 to $25 \mathrm{~cm}$ with average size of $7.3 \mathrm{~cm}$. Lymph node metastases were positive in 12 patients and negative in 42 patients. 48 out of 54 patients received any surgical treatments including lobectomy in 35 patients, pneumonectomy in 8 patients, and other resection in 5 patients. The 2-year, 5 -year, and 10-year overall survival rates were $46.4,40.2$, and $34.5 \%$, respectively (Fig. 3a). The 5 -year overall survival rates in no lymph node metastatic group and lymph node metastatic 
a

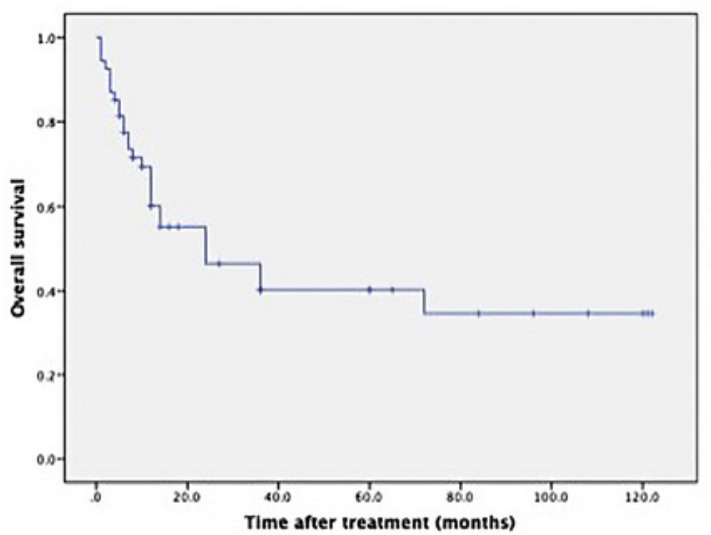

b

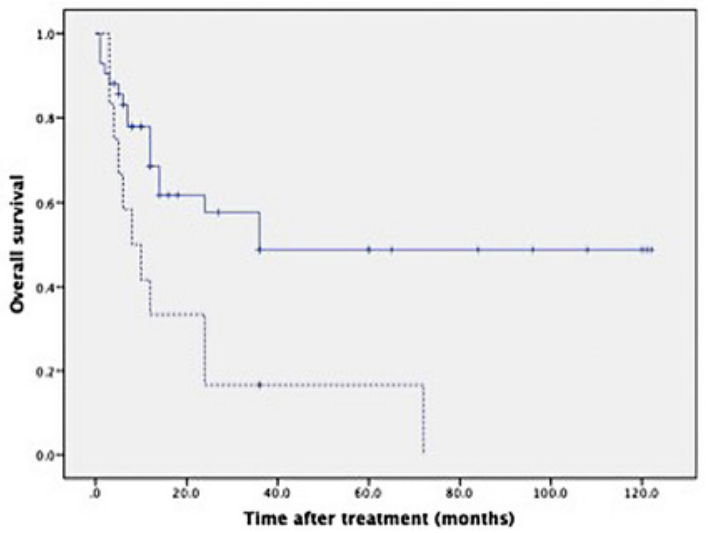

Fig. 3. The 2-year, 5-year, and 10-year overall survival (OS) rates were $46.4 \%, 40.2 \%$, and 34.5\%, respectively (a). The 5-year OS rates in no lymph node metastatic group (solid line) and lymph node metastatic group (dotted line) were 48.7 and $16.7 \%$, respectively, with a significant difference $(p=0.006)(\mathbf{b})$.

group were 48.7 and $16.7 \%$, respectively, with a significant difference ( $p=0.006$ ) (Fig. 3b). According to these data, nodal status might contribute to the prognosis of primary pulmonary UPS/MFH as well as lung cancer. The effective treatment for UPS/MFH is complete resection and appropriate surgical procedure is lobectomy.

Few reports have evaluated the effectiveness of chemotherapy, including combination chemotherapy with cyclophosphamide, vincristine, adriamycin, and dacarbazine [9]. Edmonson et al. [10] reported that combination chemotherapy using doxorubicin and ifosfamide improved the response rate and progression-free survival. However, consensus regarding standard treatment for primary pulmonary UPS/MFH has not been established. Although chemotherapy for UPS/MFH is in generally not a promising treatment modality, our patient achieved long-term complete response in accordance with Edmonson's report [10]. This case is encouraging regarding patients with UPS/MFH; however, we will continue to follow our patient, closely. Doxorubicin and ifosfamide treatment may be more likely to cause myelosuppression [10] compared with doxorubicin alone, and our patient suffered grade 3 myelosuppression and subsequent febrile neutropenia. Physicians must collect and evaluate data describing both the effectiveness and adverse events of multidisciplinary treatment for this rare entity.

In non-small cell lung cancer, major advances have been made in treatment with the advent of immune-checkpoint inhibitors such as nivolumab (anti-programmed cell death 1 [PD-1] antibody), pembrolizumab (anti-PD-1 antibody), durvalumab (anti-PD-L1 antibody), atezolizumab (anti-PD-L1 antibody), and ipilimumab (anti-cytotoxic T lymphocyte antigen 4 antibody). In patients with advanced bone and soft tissue sarcomas, pembrolizumab showed promising activity in the SARC028 trial [11]. In this trial, responses to pembrolizumab were seen even in the absence of PD-L1 expression; however, the authors stated that the role of PD-L1 expression in soft-tissue sarcoma remains unclear. Based on this trial, a novel phase II study is now ongoing that is evaluating single-agent anti-PD-1 antibody and combination anti-cytotoxic T lymphocyte antigen 4 (CTLA-4) and anti-PD-1 therapy for surgically resectable UPS/MFH and dedifferentiated liposarcoma [12]. We expect these immunotherapies will be promising treatment modalities for UPS/MFH in the near future; however, a specific biomarker is needed to predict the effectiveness and prognosis of immune checkpoint inhibitors in this rare sarcoma. 
Higuchi et al.: Successful Treatment for Primary Pulmonary Undifferentiated

Pleomorphic Sarcoma

UPS/MFH is reported to have genetic mutations, which are responsible for primary pulmonary UPS/MFH formation and progression. Li et al. [13] reported that the mutation frequency of the tuberous sclerosis complex 2 gene was $15.64 \%$, and this gene activates the mammalian target of rapamycin (mTOR) pathway. Li et al. [5] reported a case of concurrent KRAS mutation and phosphatidylinositol 3-kinase p110 subunit alpha (PIK3CA) mutation [8]. Serrano et al. [14] also reported that the RAS/mitogen activated protein kinase (RAS/MAPK) and phosphatidylinositol 3-kinase (PI3K)/mTOR pathways were activated in the majority of patients with UPS/MFH. KRAS is a proto-oncogene located at $12 \mathrm{p} 12.1$, and a frequently altered gene, with mutations occurring in 17-25\% of all cancers [5]. Constitutive activation of growth factor signaling pathways is responsible for the maintenance of aggressiveness and tumor phenotype, and among the pathways, the RAS/MAPK and PI3K/mTOR pathways commonly drive oncogenic stimuli in soft tissue sarcomas [15]. Serrano et al. [14] reported that RAS/MAPK was activated in the majority of patients with UPS, and that this pathway contributed to the aggressive behavior of UPS/MFH. Considering these findings, our patient's medical status was dire, both before and after surgery. The clinical investigation of novel agents targeting the RAS/MAPK pathway in UPS/MFH as well as in other malignancies, is urgently needed.

\section{Conclusions}

Our patient with primary pulmonary UPS/MFH achieved long-term complete response following multidisciplinary treatment even though this sarcoma carries a poor prognosis and is insensitive to both chemotherapy and radiotherapy.

\section{Acknowledgements}

The authors thank Mr Takashi Sato, Ms Miho Sagawa, and Ms Michie Kojimahara from the Department of Pathology, Aizu Medical Center, Fukushima Medical University for their excellent technical work and support, and Dr Jane Charbonneau, DVM, from Edanz Group (www.edanzediting.com/ac) for editing a draft of the manuscript.

\section{Statement of Ethics}

The need for ethical approval was waived for this case report. Written consent was obtained from the patient to undergo the procedures described in this report. Written consent was obtained from the patient for the publication of this case report and accompanying images.

\section{Disclosure Statement}

The authors have no conflicts of interest to declare.

\section{Funding Sources}

This research did not receive any specific grant from funding agencies in the public, commercial, or not-for-profit sectors. 


\section{Author Contributions}

M.H. collected and assembled the data, and drafted the article. H.Y., K.M., I.O., N.S., T.S., H.U., S.Y., and H.H. assisted with data collection. H.S. helped draft the article and finally approved the article. All authors read and approved the final manuscript.

\section{References}

1 O'Brien JE, Stout AP. Malignant fibrous xanthomas. Cancer. 1964;17:1445-55.

2 Weiss SW, Enzinger FM. Malignant fibrous histiocytoma: an analysis of 200 cases. Cancer. 1978;41(6):225066.

3 Fletcher CD. The evolving classification of soft tissue tumours - an update based on the new 2013 WHO classification. Histopathology. 2014;64(1):2-11.

4 Maitani F, Fujimori S, Hayashi Y, Hasegawa A, Iwazaki M. A case of juvenile primary pulmonary malignant fibrous histiocytoma. Tokai J Exp Clin Med. 2010;35(4):130-2.

5 Li B, Li L, Li X, Wang Y, Xie Y, Liu C, et al. Undifferentiated pleomorphic sarcoma with co-existence of KRAS/ PIK3CA mutations. Int J Clin Exp Pathol. 2015;8(7):8563-7.

6 Qorbani A, Nelson SD. Primary pulmonary undifferentiated pleomorphic sarcoma (PPUPS). Autops Case Rep 2019 Jul-Sep; 9(3):e2019110.

7 Pleština S, Librenjak N, Marušić A, Batelja Vuletić L, Janevski Z, Jakopović M. An extremely rare primary sarcoma of the lung with peritoneal and small bowel metastases: a case report. World J Surg Oncol 2019;17: 147.

8 Coșgun T, Tezel Y, Akyıl M, Kolbaș İ, Șen A, Tezel Ç. Primary pulmonary malignant fibrous histiocytoma. Turk Thorac J 2017;18:54-6.

9 Leite C, Goodwin JW, Sinkovics JG, Baker LH, Benjamen R. Chemotherapy of malignant fibrous histiocytoma: a Southwest Oncology Group report. Cancer. 1977;40(5):2010-4.

10 Edmonson JH, Ryan LM, Blum RH, Brooks JS, Shiraki M, Frytak S, et al. Randomized comparison of doxorubicin alone versus ifosfamide plus doxorubicin or mitomycin, doxorubicin, and cisplatin against advanced soft tissue sarcomas. J Clin Oncol. 1993;11(7):1269-75.

11 Tawbi HA, Burgess M, Bolejack V, Van Tine BA, Schuetze SM, Hu J, et al. Pembrolizumab in advanced soft-tissue sarcoma and bone sarcoma (SARC028): a multicentre, two-cohort, single-arm, open-label, phase 2 trial. Lancet Oncol. 2017;18:1493-501.

12 Keung EZ, Lazar AJ, Torres KE, Wang WL, Cormier JN, Ashleigh Guadagnolo B, et al. Phase II study of neoadjuvant checkpoint blockade in patients with surgically resectable undifferentiated pleomorphic sarcoma and dedifferentiated liposarcoma. BMC Cancer. 2018;18(1):913.

13 Li X, Liu R, Shi T, Dong S, Ren F, Yang F, et al. Primary pulmonary malignant fibrous histiocytoma: case report and literature review. J Thorac Dis. 2017;9(8):E702-8.

14 Serrano C, Romagosa C, Hernández-Losa J, Simonetti S, Valverde C, Moliné T, et al. RAS/MAPK pathway hyperactivation determines poor prognosis in undifferentiated pleomorphic sarcomas. Cancer. 2016;122(1): 99-107.

15 Dodd RD, Mito JK, Eward WC, Chitalia R, Sachdeva M, Ma Y, et al. NF1 deletion generates multiple subtypes of soft-tissue sarcoma that respond to MEK inhibition. Mol Cancer Ther. 2013;12(9):1906-17. 